dem $\mathrm{Z}$ ells t of f unserer Laub- und Nadelholzbäume hergestellten Papiere. In Festigkeit stehen sie den Hadernpapieren wenig oder gar nicht nach, wohl aber in Lebensdauer, Lichtbeständigkeit und in gewissen Grenzen auch in Druckfähigkeit. Eine eigenartige glasige Sprödigkeit und mit dem Alter zunehmende Brüchigkeit ist allen Zellstoffpapieren eigen. Je nach A r t, Dauer und Ausfall der Zellstoffkochung, Gründlichkeit bei der Auswaschung der Laugenreste und Reinigung von Knoten, unaufgeschlossenen Faserbündeln, verkohlten Holzresten, Grad der Bleichung usw. ist der Wert der Zellstoffe ein außerordentlich verschiedener und demgemä $\beta$ auch der Wert der hieraus gefertigten Papiere. Ohne Zusatz von weißfärbenden Mineralien, welche die knöcherne Sprödigkeit und die Transparenz mildern, würden die Zellstoffpapiere kein befriedigendes Druckresultat geben können. Namentlich für Illustrationsdruckpapiere ist ein größerer Zusatz von Tonerde (China clay) unerläßlich.

Bei den holzschliffhaltigen Papieren tritt die Brïchigkeit und die Neigung zum Vergilben am meisten in Erscheinung. Dies erklärt sich daraus, weil die Zerfaserung des hierzu verwendeten Holzes nicht wie beim Celluloseprozeß durch Kochen in Laugen, sondern auf dem viel billigeren Wege des mechanischen Abschleifens auf rotierenden Sandsteinen erfolgt. - Während beim Cellulose $\mathrm{k} \mathrm{o} \mathrm{c}$ prozeß die Ursachen des Vergilbens, nämlich alle Harze und die Inkrusten somit die eigentlichen Holzstoffe in die Kochlauge gelöst übergingen und mit dieser entfernt wurden, wobei die reine Cellulose übrig blieb, gehen beim $S \mathrm{c} \mathrm{h} \mathrm{le} \mathrm{if} \mathrm{-}$ proze $B$ alle vergilbenden Lignitstoffe und Harze in das Schleifgut mit über; sie bleiben an den Zellstofftrümmern hängen und gelangen mit diesen gemeinschaftlich in das hieraus gefertigte Papier. Je mehr Holzschliff nun in einem Papier enthalten ist, je gröber der Schliff ausfiel, und je lignitreicher und dabei zellstoffärmer das zerschliffene Holz gewesen ist, desto rascher und nachhaltiger wird das betreffende Papier vergilben. Die Brüchigkeit der holzsehliffhaltigen Papiere hängt mit der ganzen Struktur der scharfkantig zertrümmerten Holzfragmente zusammen. Es fehlen hier die feinen und feinsten Fäserchen (Fibrillen), welche allein eine innige Verfilzung ermöglichen können. Durch Wahl zellstoffreicher Hölzer und eigenartige Schleifmethoden ist es in neuester Zeit zwar gelungen, auch den durch $\mathrm{S} \mathrm{ch}$ le if en abgetrennten Holzfasern eine gewisse Länge und hiermit eine größere Haltbarkeit zu geben. Die hieraus gefertigten Papiere haben eine durchweg befriedigende Festigkeit, Vergilben weniger rasch, namentlich bei Zusatz von ca. $50 \%$ Cellulose und $10-15 \%$ Hadern, und drucken sich bei entsprechender Glätte vorzüglich, namentlich für Autotypien. Sie stehen den ganz billigen holzfreien, nur aus Zellstoff gearbeiteten Papieren weder in Färbung, noch Festigkeit viel nach, drucken sich aber besser und sind dabei um ca. $10 \%$ billiger. Unsere mit Massenauflagen rechnenden illustrierten Zeitschriften und Familienjournale sind auf Papiere dieser Stoffmischung gedruckt, ebenso die annähernde Hälfte der im Verlagsbuchhandel erscheinenden Werke.

$\mathrm{Da}$ es technisch unmöglich ist, aus brüchigen
Rohstoffen (z. B. aus Holzschliff, aus stark gechlortem Zellstoff, aus mürben, abgetragenen Hadern, aus Fangstoffen usw.) ein festes Papier herzustellen, so hat man von jeher den Kaufwert eines Papiers in erster Linie nach seiner Fes t ig k e it und nach seiner reinen $G \mathrm{r}$ u $\mathrm{n} d \mathrm{w}$ e i $\mathrm{B}$ e beurteilt. Man hält zu diesem Zweck das betreffende Blatt gegen das durchfallende Licht, wobei alle Unreinheiten und ferner die Menge des mineralischen Füllstoffs an Weißerde beurteilt werden können. Je mehr das Papier mit Gips, Ton u. dgl. gefüllt wurde, desto undurchsichtiger ist es. Auch Holzsehliff macht das Papier undurchscheinend. Gute, holzfreie, wenig beschwerte Papiere haben eine lichte, durchscheinende Durchsicht. Reißen wir das betreffende Papierblatt vorsichtig in der Längsund in der Querrichtung ein, so wird ein festes Papier viel Widerstand bieten und einen langfaserigen $\mathrm{RiB}$ aufweisen, ein haltloses Papier dagegen fast von selber auseinander fallen und im RiB nur vereinzelte kurze Fasern zeigen.

Auf sehr sinnreich konstruierten Zerreiß. maschinen läßt sich die Festigkeit eines Papiers durch Zahlenwerte ganz genau ermitteln, seine Elastizität und sein Widerstand gegen Reiben und Knittern durch trockene Wäsche zwischen den Handballen. Holzschliff läßt sich durch Phloroglucin, Anilinsulfat und eine Reihe anderer Reagenzien leicht nachweisen, die Fasermischung eines Papiers aber durch Abschätzung unter dem Mikroskop. Der Aschengehalt wird durch Verbrennen und WeiBglühen eines bestimmten Gewichtsquantums Papier und Abwägen des Aschenrückstandes genau festgestellt; die Ermittlung freier Mineralsäuren erfolgt durch bekannte säureempfindliche Indicatoren, zu denen blaues Lackmuspapier aber nicht gezählt werden kann, da von der Harzleimung herrührende Tonerdesalze im Papier vorhanden sein können, durch welche ebenfalls eine Rotfärbung des Lackmuspapiors eintritt. Die quantitative Ermittlung der gefundenen Säure hat wenig Zweck, da Spuren ausreichen, einen vorzeitigen Zerfall des Papiers herbeizuführen oder bunte Druckfarben in der Nuance nachteilig zu beeinflussen. Die Art der Benutzung und der Ort der Aufbewahrung eines Buches trägt viel zur Fìnaltung oder Vernichtung desselben bei. In der Nähe von Fabrikschloten, ferner in feucht-nebeligen Distrikten können und werden sich unsere Bücher nicht ebenso gut erhalten, wie in trockener und reiner Luft; wechselnde Trockenheit mit Feuchtigkeit führt aber selbst die besten Papiere bald zum Zerfall.

\section{Die Größenbestimmung der Fragmente des Kakaopulvers.}

\author{
Von EDM. Hoppe. \\ (Eingeg. den 29./4, 1908.)
}

In gegebener Veranlassung war ich genötigt, die Größe der kleinen Teilchen im Kakaopulver zu bestimmen. Messungen über diese Größen hat, soviel ich weiß, Prof. H u e p p e (Prag) 1905 veroffentlicht, in welchen er die im Mikroskop ge- 
messenen Längen und Breiten für eine Reihe von Kakaosorten angibt. Ferner sind die Mikrophotogramme von $\mathrm{N}$ e u m a $\mathbf{n} \mathbf{n}$ in seiner Arbeit „Die Bewertung des Kakaos" veröffentlicht worden. Aus beiden ist das Volumen dieser Fragmente nicht zu bestimmen. Wenn man einen Objektträger mit Kakaopulver in atmosphärischer Luft bestäubt, sieht man sofort bei hinreichender Vergrößerung, daß die kleinen Teile vielfach aneinander kleben und dadurch sehr viel größer erscheinen, als sie in Wahrheit sind. Schon bessere Resultate erhält man, wenn das Pulver in einen mit trockener Luft gefüllten Rezipienten gebracht und durch einen trockenen Luftstrom zerstäubt wird. Ein in solchem Rezipienten niedergelegter Objektträger zeigte eine außerordentlich gleichmäßige Bestäubung, und die einzelnen Fragmente erschienen besser isoliert. Dennoch klebten auch hier noch viele Partikeln aneinander, was bei dem teilweisen Utberlagern der Fragmente durch die dunklere Färbung wahrscheinlich wurde. Da außerdem die größeren Fragmente zweifellos sehr flach waren, wie man wegen der Durchsichtigkeit und der Struktur schließen mußte und naturgemä $\beta$ stets auf der breiten Seite liegen, war durch solche Bestäubung, selbst wenn man durch weitere Austrocknung das Kleben vermeiden könnte, keine Beantwortung der Frage, wie groß ihr Volum sei zu erwarten, solange man kein Mittel fand, die flachen Gebilde unter dem Mikroskop auf die schmale Seite zu stellen, um so auch die dritte Dimension bestimmen zu können. Als solches Mittel bot sich die Suspension des Pulvers in einer Flüssigkeit dar. Als einzige Flüssigkeit konnte wohl nur Ôl in Frage kommen, da in diesem die Lösung der löslichen Substanzen sehr langsam erfolgt.

Am geeignetsten fand ich Cedernöl; bringt man einen kleinen Tropfen auf den Objektträger, stäubt etwas Pulver darauf, so legt sich dasselbe zunächst auf die Oberfläche, und da diese nahezu ein Ellipsoid. ist, kann man nach dem Rande zu schon einige Fragmente finden, welche dem Gesichtsfelde des Mikroskops die schmale Seite zukehren oder doch so schräg liegen, daß man diese dritte Dimension messen kann. Immerhin ist diese Messung nur vereinzelt möglich und bietet daher keine Gewähr, Durchschnittswerte zu bekommen. Durch mechanische Erschütterung kann man die zunächst auf der Oberfläche liegenden Staubteilchen in dem Tropfen selbst suspendieren. Hierbei zeigt sich, wie die aneinanderklebenden Teile sich trennen und nun einzeln in dem Öl schweben. Allein die Hoffnung, daß dio Teilchen in allen möglichen Lagen sich darbieten würden, erfüllte sich in bezug auf die größeren Fragmente nicht, sie lagen in ganz überwiegender Zahl mit der Breitseite vor dem Linsensystem. Wenn man nun aber einen feinen Kupferdraht unter dem Tropfen an den Objektträger bringt oder auch seitlich vom Tropfen von oben her in die Nähe des Öles bringt und das freie Ende dieses Drahtes in eine Bunsenflamme steckt, so wird von der Spitze des Drahtes aus das Öl erwärmt, es entstehen in dem Tropfen Strömungen die kleinen suspendierten Fragmente werden herumgedreht, und man kann bei einer großen Zahl auch ziemlich breiter Fragmente die dritte Dimension messen. Die größten Stücke verschieben sich freilich in der Regel ihrer ursprünglichen Lage parallel ohne Drehung, doch stimmen die vereinzelten Messungen der dritten Dimension an solchen großen Platten mit den vielfachen Messungen an kleineren so gut überein, daß kein Grund vorhanden ist, für dje dritte Dimension nicht einen allgemeinen Mittelwert zu bilden. Die Messungen sind so lange fortzusetzen, als die unveränderte Farbe des Öls zeigt, daß durch Lösung noch keine Größenveränderung eingetreten ist. Erst nach geraumer Zeit färbt sich das Öl, und dann ist natürlich keine Messung mehr zulässig.

Die Messungen erstreckten sich auf das in Würfeln gepreßte Kakaopulver der Marke Afrikaner von der Reichard-Compagnie. Folgende Tabelle ist ein Auszug aus den Beobachtungen.

\begin{tabular}{|c|c|c|c|}
\hline Fragmente & $\begin{array}{l}\text { Ocular } \\
\text { skala }\end{array}$ & $\underset{u}{\text { Wert in }}$ & $\begin{array}{c}\text { Mittel aus allen } \\
\text { Messungen } \\
\text { in } \mu\end{array}$ \\
\hline $\begin{array}{c}\text { Länge maxim. . . . } \\
\text { ", minim. . . }\end{array}$ & $\begin{array}{r}22 \\
6\end{array}$ & $\begin{array}{l}38,1 \\
10,4\end{array}$ & 23,0 \\
\hline $\begin{array}{c}\text { Breite maxim. . . } \\
\text {, } \quad \text { minim. . . }\end{array}$ & $\begin{array}{c}12 \\
5,1\end{array}$ & $\begin{array}{r}20,1 \\
8,8\end{array}$ & 10,13 \\
\hline $\begin{array}{c}\text { Dicke maxim. . . . } \\
,, \quad \text { minim. . . }\end{array}$ & $\begin{array}{l}2,3 \\
1,3\end{array}$ & $\begin{array}{l}4,0 \\
2,2\end{array}$ & 3.0 \\
\hline
\end{tabular}

Daraus ergibt sich, $\mathrm{da} \beta$ in Mittel in einem Kubikzentimeter des zusammengepreBten Pulvers 1430. $10^{6}$ kleine Teilchen vorhanden sind, und wenn man nur die größten Teilchen zur Berechnung des Volumens zuläBt, würde $1 \mathrm{ccm} 326.10^{6}$ Teilchen enthalten.

\section{Die Ausnutzung der Wasserkräfte Skandinaviens.}

\author{
Von Fritz Kruld, Muttenz (Schweiz). \\ (Eingeg. 9./4. 1908.)
}

Die Ausnutzung der Wasserkräfte Skandinaviens ist geit 10 Jahren in leb. haftester Entwicklung begriffen, besonders im Süden der Halbinsel. Hauptsächlich sind es für Nor w e g e $\mathbf{n}$ die Wasserkräfte des G lo m m e $\mathbf{n}$ flusses, des Skienflusses und des Drammenflusses und für Schweden die Wasserkräfte des Götaflusses bei Trollhättan und des Laganflusses in seinem Unterlaufe. Norwegen besitzt in seinen Flußläufen bei mittlerem Wasserstande in ganzen etwa 30 Mill. PS, von denen etwa 4 Mill. PS. ohne weiteres ausnutzbar sind; $1^{1 / 4}$ Mill. davon liegen im südlichen Norwegen. Von diesen werden zurzeit etwa 250000 PS verwertet. Die Kosten für den Ausbau dieser 4 Mill. PS sind außerordentlich gering und betragen für 1 PS einschließlich der elektrischen Übertragung 250-350 M, und die Jahresausgaben sind für eine PS $30-40 \mathrm{M}$, in einigen besonders günstigen Fällen sogar nur $10 \mathrm{M}$ und weniger. Hierbei sind die im Zuge der Flüsse liegenden natürlichen Seen von bedeutendem Nutzen, da sie mit verhältnismäßig niedrigen Kosten in gewaltige 